\title{
EXCLUSION-ZONE FORMATION FROM DISCONTINUOUS NAFION SURFACES
}

\author{
XAVIER A. FIGUEROA \& GERALD H. POLLACK \\ Department of Bioengineering, Box 355061, University of Washington, Seattle WA, 98195.
}

\begin{abstract}
Many hydrophilic materials in aqueous solution show near-surface zones that exclude suspended colloids and dissolved molecules. These exclusion zones (EZs) can extend for tens to hundreds of micrometers from the hydrophilic surface, and show physicochemical properties that differ from bulk water. So far, only continuous surfaces of polymers, gels, or biological specimens have been studied. In this report, we explore the interactions between exclusion zones generated by discontinuous, regularly spaced EZ-generating surfaces, namely strips of Nafion on a glass surface. Various inter-strip spacings were studied. When Nafion surfaces are separated by $100 \mu \mathrm{m}$ or less, EZs merged with one another, forming a single, continuous, stable EZ. Separations larger than $100 \mu \mathrm{m}$ produced discontinuous EZs that did not merge. This result has implication for the mechanism by which independent EZs can merge with one another.

Keywords: exclusion zone, microspheres, Nafion, photolithography, unstirred water layer, water, water ordering, water structure.
\end{abstract}

\section{INTRODUCTION}

When water interacts with hydrophilic surfaces, the interfacial water molecules reorder. Water ordering is commonly thought to be limited to a few water-molecular layers and to have limited macroscopic effects. Yet, recent studies on physical properties of interfacial water suggest that this may not be the case and important new features of interaction of liquid water at hydrophilic surfaces are coming to be understood.

Over the last century, evidence has shown interfacial water at many hydrophilic surfaces to be physically $[1,2]$ and electrically $[3,4]$ distinct from bulk water. More recent evidence $[5,6]$ reveals that these exclusion zones represent a state of water that is more ordered than bulk water. The ordering is hypothesized to produce the observed exclusion, as well as the electric potential and $\mathrm{pH}$ gradients. Such features of the exclusion zones (EZs) offer promising applications such as water purification [7], mechanical sorting [5, 8-10], power storage [4], and potentially new waterbased chemistries that take advantage of the properties of interfacial water in enzyme-assisted reactions [11].

Although the mechanism underlying EZ formation is not fully resolved, EZs can be readily generated and observed. Of interest is determining the ability of one EZ to interact with another, as any such information sheds light on the EZ's basic structural and chemical features. This in turn could provide a better understanding of related phenomena in biology, such as the unstirred layer [12] and various structural changes in tissues [13].

Here we report that patterned Nafion on a glass surface produces EZs that can merge with each other and that this merging is dependent on the spacing between the Nafion sections. Results show that when the Nafion strips are more than $100 \mu \mathrm{m}$ apart, the respective EZs remain separate; conversely, when the strips are separated by less than $100 \mu \mathrm{m}$, the neighboring EZs merge to form a single, continuous, exclusion zone. 


\section{MATERIALS AND METHODS}

\subsection{General outline}

Briefly, microfluidic devices are used as stencils for depositing liquid Nafion in specific patterns onto glass coverslips (Fig. 1). The liquid is allowed to dry on the coverslip, and sections of the glass/ Nafion coverslip are then cut for subsequent implementation.

Implementation is shown in Fig. 2. An enclosed chamber (made of clear PDMS rubber) is placed over the vertically positioned glass section containing the Nafion strips; vertical placement facilitates viewing.

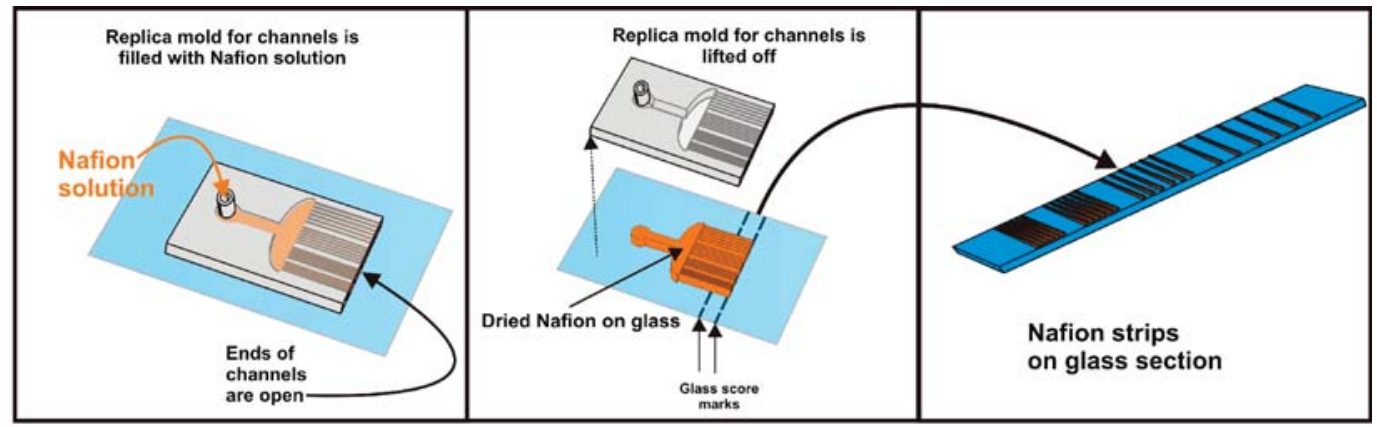

Figure 1: Nafion strip deposition onto glass. Left, a PDMS microfluidic device is placed on top of a glass coverslip. Nafion solution is injected via an entry port and filled. Center, Nafion is allowed to dry inside the microfluidic device. The microfluidic device is removed and the Nafion strips are left on the glass coverslip. Right, the glass coverslip is scored on the backside with a carbide pen. Sections of the glass coverslip containing the deposited Nafion strips are removed.

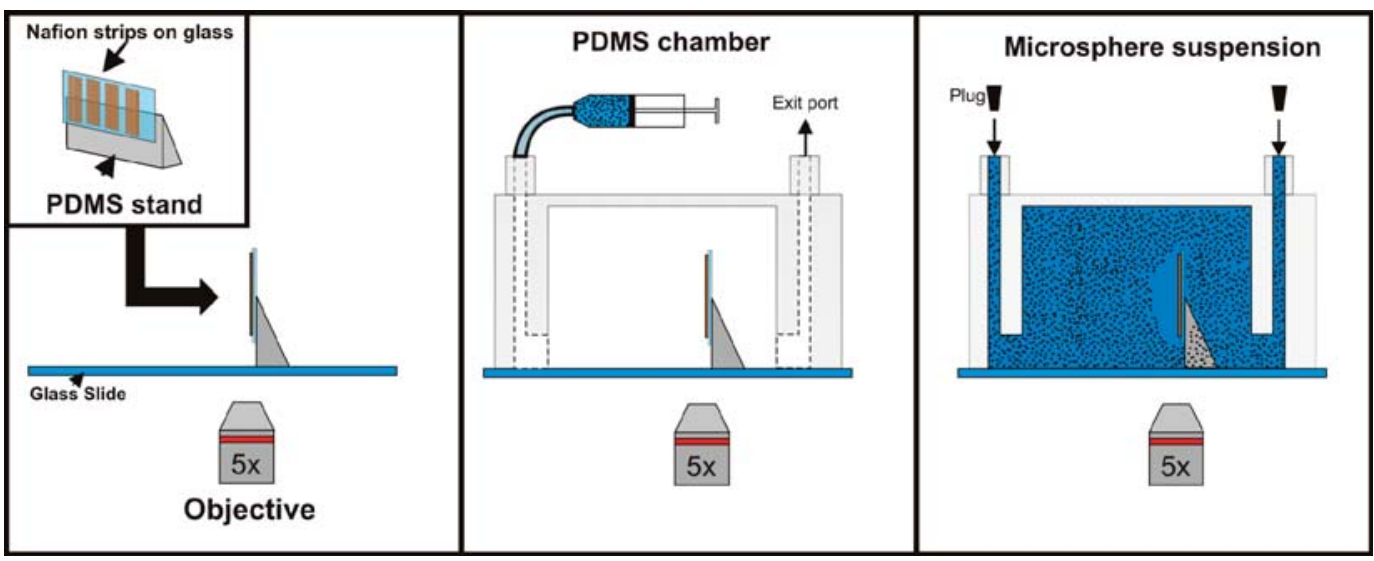

Figure 2: Experimental setup for EZ observation. Left, a glass section with Nafion strips is attached to a PDMS stand. The stand with the Nafion strips is placed on a glass slide. Center, a PDMS chamber is placed over the stand + strips and a syringe containing water is used to fill the chamber. Once the Nafion strips are allowed to hydrate, the microsphere solution is delivered into the chamber. Right, the chamber is sealed with plugs and images of EZ formation are acquired. 
An aqueous suspension of microspheres is then delivered by a tube connected to a $10 \mathrm{~mL}$ syringe, to fill the PDMS chamber. After delivery, both ports are plugged to minimize any flow arising from fluid-level imbalances on either side. Images of Nafion-generated EZs are then taken in a time series.

\subsubsection{Production of a master mold}

For constructing the microfluidic device, production of the master mold is an essential first step. Master molds with micron-scale resolution are raised features that sit on a flat surface (usually a silicon wafer). They are generated by spreading a thin uniform layer of light-sensitive polymer onto the wafer, and exposing it to UV light. The light initiates a chemical reaction that cross-links, and thereby hardens, the polymer. By shining the UV light through a UV-opaque patterned mask, a corresponding pattern of raised features can be produced. The uncrosslinked areas are later removed by immersion in a solvent (SU-8 developer; MicroChem Corp.) with agitation for several minutes. A detailed protocol for generating master molds is discussed elsewhere [14].

\subsubsection{Production of microfluidic device}

The raised features of the master mold are next copied as a reverse cast in polydimethylsiloxane (PDMS). PDMS comes as a liquid pre-polymer; when mixed with a catalyst it hardens into a pliable, clear solid, excellent for optical imaging. Briefly, PDMS was mixed with the catalyst. Bubbles were removed by placing the mixture in a partial vacuum. The PDMS was then poured directly on top of the master mold and incubated for 2 hours at $80^{\circ} \mathrm{C}$ to accelerate the curing reaction. Once cured, the replicated features were peeled off the surface of the master mold. The reversed features, copied onto the PDMS, were used as channels for deposition the liquid Nafion onto the glass substrate (Fig. 1). Multiple replicas were made from a single master mold.

\subsubsection{Nafion deposition}

The Nafion solution was deposited onto the glass coverslip in two different patterns, each using a different method. For the first pattern, used as control, a continuous, even coat of Nafion solution was applied to the glass coverslip using an airbrush (Paasche Professional DOUBLE-Action Airbrush Set, Model\# VL-SET, Paasche Airbrush Company, Chicago, Illinois). The sprayed-on Nafion layer was dried on the coverslip for $2 \mathrm{hrs}$ at $80^{\circ} \mathrm{C}$ and stored at room temperature for use within one week. In the second method (Fig. 1), strips of Nafion were generated on a glass coverslip by injecting Nafion solution into the microfluidic device. The strips were dried overnight at room temperature to ensure even deposition. The PDMS film was peeled off the glass coverslip, leaving behind the Nafion strips.

The Nafion strips were deposited as four distinct patterns with set distances between the strips. The spacing between edges of strips were set respectively at $50 \mu \mathrm{m}, 100 \mu \mathrm{m}, 250 \mu \mathrm{m}$, and $500 \mu \mathrm{m}$. Isolated strips were set at 2-mm distances from each other. This distance was assumed to be large enough that nearby strips could be studied in isolation.

\subsubsection{Microsphere suspensions and visualization chambers}

Microsphere suspensions were made in $15 \mathrm{~mL}$ tubes and kept in the dark when not in use. The suspensions were made of $2-\mu \mathrm{m}$ diameter carboxylate-conjugated polystyrene microspheres (Polyscience, Inc.; Warrington, PA), diluted to $\sim 8.5 \times 10^{6}$ microspheres $/ \mathrm{mL}$. The $\mathrm{pH}$, measured prior to each experiment, was 7.7-8.2, and fresh suspensions were made weekly. All suspensions were made with distilled, deionized water (NanoPure Diamond, Barnstead, 18.3 M $\Omega$ ).

Small, enclosed PDMS chambers with a clear viewing window on the top were designed to contain the glass sections with Nafion (Fig. 2). The Nafion strips on glass were held vertically using a 
rectangular PDMS stand, allowing for a clear viewing area. PDMS is a hydrophilic material that adheres to glass strongly, making water-tight seals without using adhesives. The PDMS chambers were enclosed to inhibit evaporative and surface air-flow-induced mixing, providing a stable environment to study EZ development.

\subsubsection{Microscopy and analysis}

All images were obtained using a Zeiss Axiovert 35 or a Zeiss Axio Observer A.1 microscope in bright-field mode (Zeiss Corporation, Heidelberg, Germany) and recorded using a CCD camera (CFW 1012; Scion Corporation, Frederick, Maryland, USA). Analysis was done using the McMaster Biophotonics Facility version of ImageJ (Wayne Rasband, National Institute of Health). All images were taken at 50x magnification.

\subsubsection{Chemicals and supplies}

Major supplies included the following: Nafion (5\% w/w perfluorosulfonic acid-PTFE copolymer, Alfa Aeser, Ward Hill, Massachusetts, USA); Silane (tridecafluoro-1,1,2,2-tetrahydrooctyl)1-trichlorosilane, (Sigma-Aldrich)); PDMS (Sylgard 184 Silicone Elastomer, Dow Corning); and, coverslip glass (Fisher, Rectangles No. 1; Size: $40 \times 22 \mathrm{~mm}$ ).

\section{RESULTS}

All the experiments described were carried out with the experimental surfaces supported vertically on a PDMS stand and situated inside a PDMS chamber (Fig. 2). All Nafion surfaces were hydrated for 5 minutes with water before replacing the water with a microsphere suspension, to expedite EZ buildup.

\subsection{Exclusion zone formation from continuous Nafion surfaces}

In these experiments, a continuous sprayed-on Nafion layer $(\sim 1 \mu \mathrm{m}$ thick) was used as a control to generate EZs. The opposite face was left untouched to act as a non-EZ forming control. Figure 3 shows the result. The thin layer of Nafion produced a relatively uniform EZ that excluded microspheres. The EZ was robust and could be generated consistently with samples that were prepared at different times. The uncoated side showed no EZ formation. The sprayed-on Nafion EZs were similar in size to EZs using Nafion-117 sheets $[5,7,15]$.

The exclusion zone grew rapidly and stabilized at $\sim 400 \mu \mathrm{m}$ within about 3 minutes. The EZs generated from these surfaces remained stable for more than an hour (Fig. 4) with only small fluctuations in the size. The uncoated backside of the coverslip never generated a visible EZ during any of the experiments. Only the Nafion-coated surface produced the exclusion zones.

\subsection{Exclusion zone formation from isolated Nafion strips}

Single Nafion strips $(20 \mu \mathrm{m}$ wide $\times 20 \mu \mathrm{m}$ high $\times \sim 800 \mu \mathrm{m}$ long) were generated as described in the Material \& Methods. Within seconds of adding the microsphere suspension, an EZ was visible near the Nafion strip as a clear zone devoid of microspheres. The strips generated stable and long-lasting exclusion zones. Microspheres were excluded almost symmetrically relative to the Nafion strips' center-line. Representative images are shown in Fig. 5.

The area of the EZ was measured by outlining the clear area where microspheres were not present. Replicate experiments showed that EZ growth rate was repeatable (Fig. 6). The EZ grew rapidly and then stabilized, maintaining an average area of $\sim 3.35 \times 10^{5} \mu \mathrm{m}^{2} \pm 1.5 \times 10^{5} \mu \mathrm{m}^{2}$. This pattern of development and stability was similar to the pattern observed with the continuous sprayed-on layer 


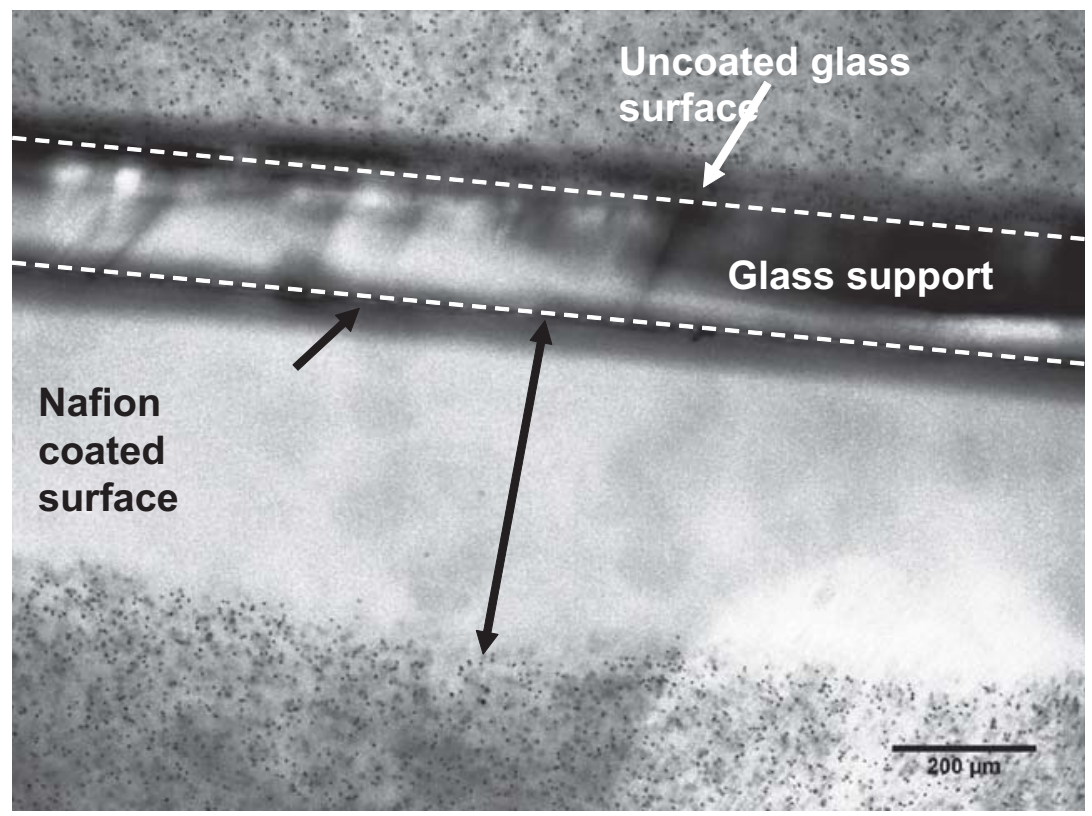

Figure 3: Microspheres ( $2 \mu \mathrm{m}$ diameter) suspended in water are excluded from the zone (EZ) adjacent to a Nafion-coated surface. The uncoated glass backside does not generate any exclusion zone. The EZ is maintained at approximately constant size for several hours. White dashed lines represent the edges of the glass support.

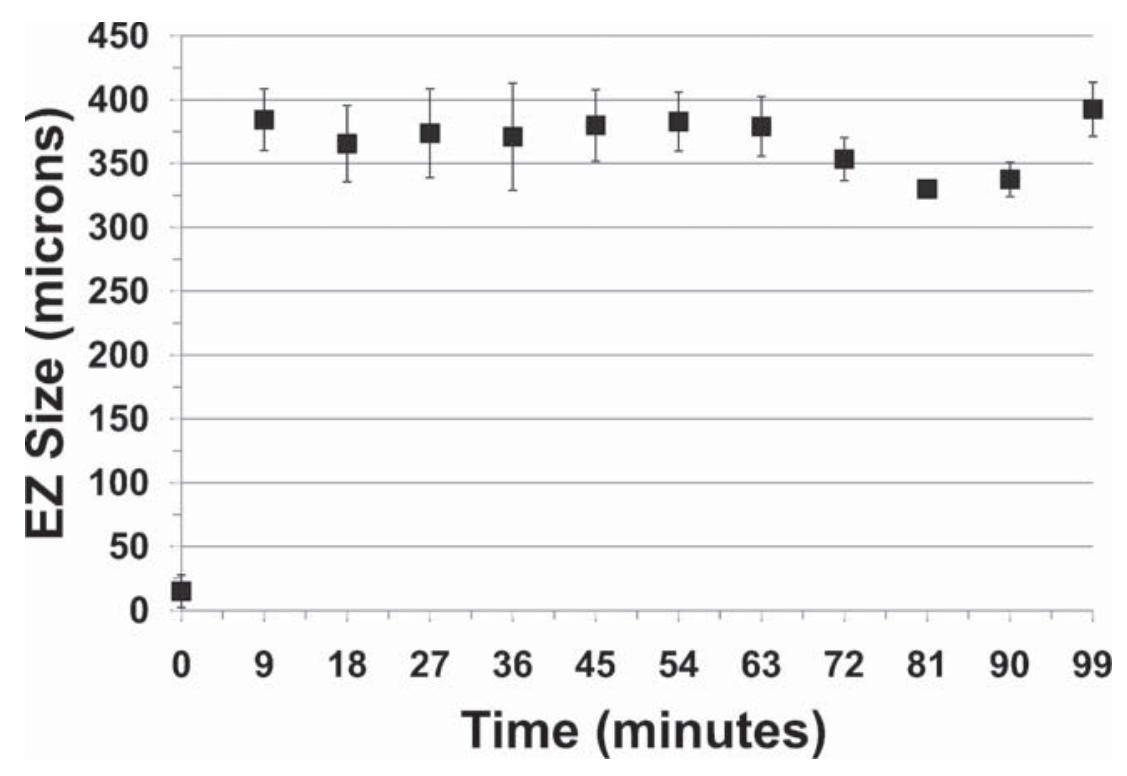

Figure 4: 


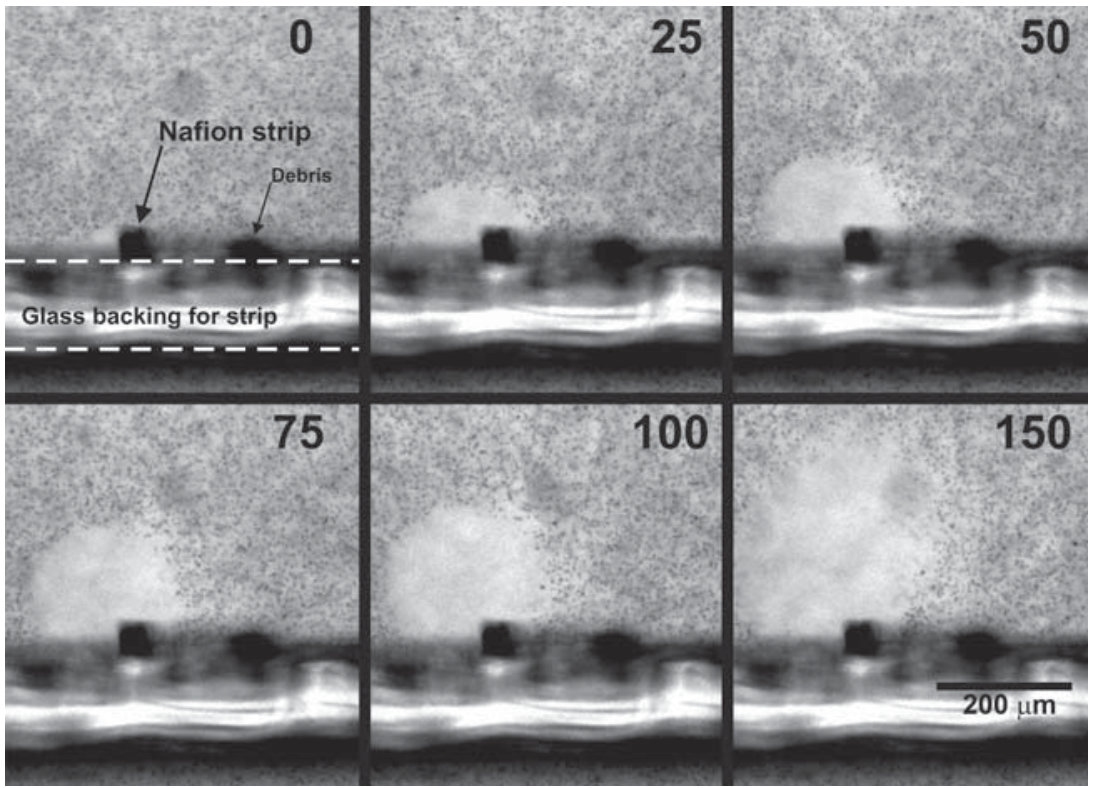

Figure 5: Exclusion-zone formation from a single Nafion strip. The Nafion strip is viewed head-on along the vertical $z$-axis. Time-lapse images were captured every five seconds. Numbers indicate time (seconds) from the start of the experiment. White dashed lines in the first panel represent the edges of the glass backing, which is out of focus.

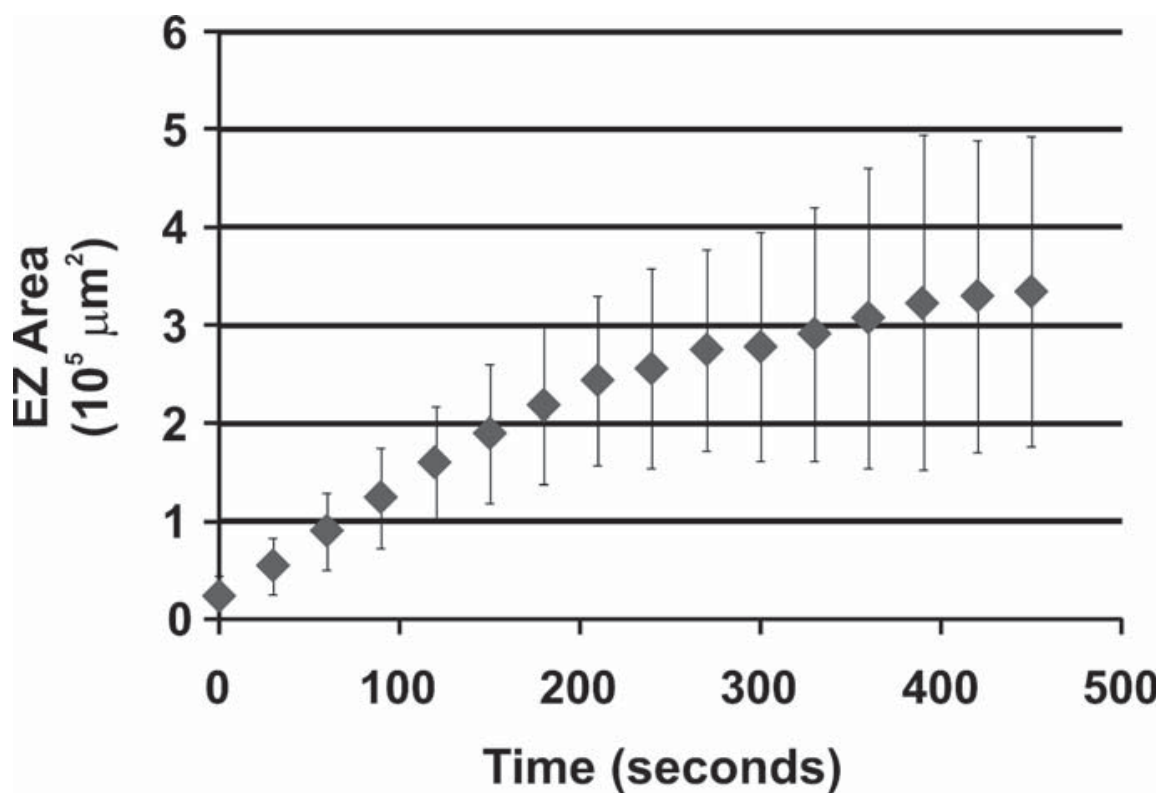

Figure 6: Area of EZ growth from an isolated Nafion strip. EZ area is defined as the region adjacent to the Nafion strip that is devoid of any microspheres. The plot is an average of data from six Nafion strips taken from three independent experiments. 
of Nafion. Differences in some important features, such as EZ width (distance from nucleating surface to outermost reaches) were apparent. In the single Nafion strips the EZs were usually narrower, and their terminal ends were unstable and constantly breaking apart, when compared to the wider and more stable EZs grown from continuous coats of Nafion.

\subsection{Effect of strip separation on EZ formation}

Given that a single Nafion strip was capable of generating an EZ, we next investigated the ability of a set of parallel strips to form a larger, continuous EZ. The strategy was to pattern groups of Nafion strips, all of the same dimension as the single strip, but differing in their edge-to-edge distances. Separations of $50 \mu \mathrm{m}, 100 \mu \mathrm{m}, 250 \mu \mathrm{m}$, and $500 \mu \mathrm{m}$ were used (Fig. 7).

A diagrammatic representation of the results is presented first to orient the reader (Fig. 8). The diagram depicts the exclusion of microspheres by Nafion strips $100 \mu \mathrm{m}$ apart, seen on face view (left), and top view at three focal depths along the $z$-axis (right). These representative results show continuous EZs in the middle region, and discontinuous ones at the tops and bottoms of the strips. Similar results were obtained with $50 \mu \mathrm{m}$ inter-strip spacing.

Actual data are shown in Fig. 9 for the full series of spacing. The groups with 50 and $100 \mu \mathrm{m}$ strip spacing produced continuous EZs in the middle sections of the strips (Fig. 9, middle row). These EZs extended $\sim 1 \mathrm{~mm}$ from the Nafion surface. Their extent and complex shape are highlighted in the top and bottom rows. At points above and below where the Nafion strips end, microspheres funnel through apparent breaks in the EZ, producing a jagged or scalloped pattern.

For the $250 \mu \mathrm{m}$ and $500 \mu \mathrm{m}$ spacing, the exclusion zones did not form a continuous EZ (Fig. 9, right columns). Instead, long, continuous columns of clear zones formed parallel to each other, centered along the Nafion strips and clearly visible in the middle row of Fig. 9. The EZs were not seen to combine, even after one hour of observation. The overall size and shape of the EZ columns remained steady, but the terminal ends of the EZs shifted, collapsed, and reformed several times, a pattern of behavior that was not seen with the $50 \mu \mathrm{m}$ and $100 \mu \mathrm{m}$ spaced Nafion strip EZs.

\section{DISCUSSION}

Previous studies of EZ formation relied on continuous surfaces to produce continuous EZs. Those surfaces included polymers, gels, and biological specimens [2]. Here, we studied discontinuous surfaces.

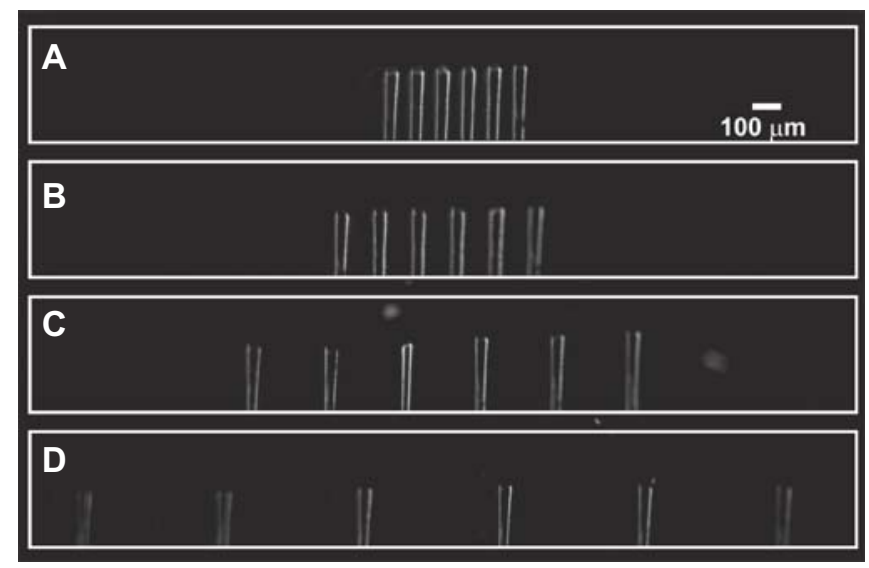

Figure 7: Deposited Nafion strips on glass, viewed from above. 


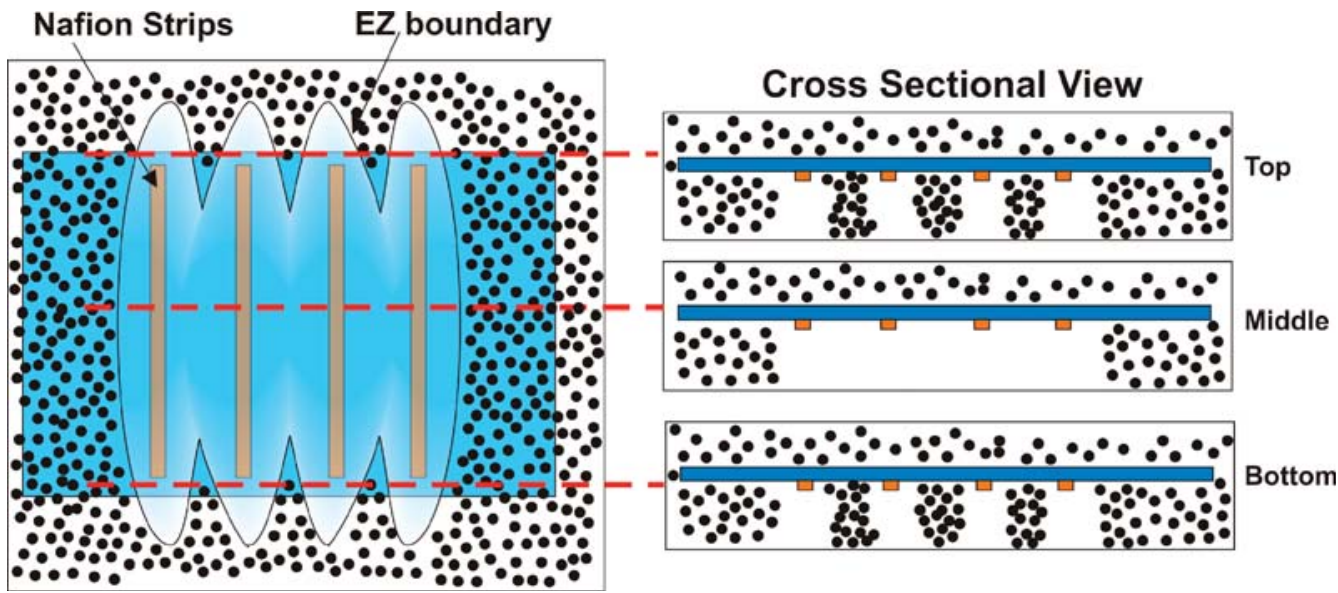

Figure 8: Diagrammatic representation of EZs obtained using $100 \mu \mathrm{m}$-separated Nafion strips. Left, face-on view. Microspheres represented by dotted areas. Clear space represents the extent of the EZ and is a reconstruction from four independent experiments. Right, EZs and microspheres as viewed from the top at three locations along vertical Nafion strips.

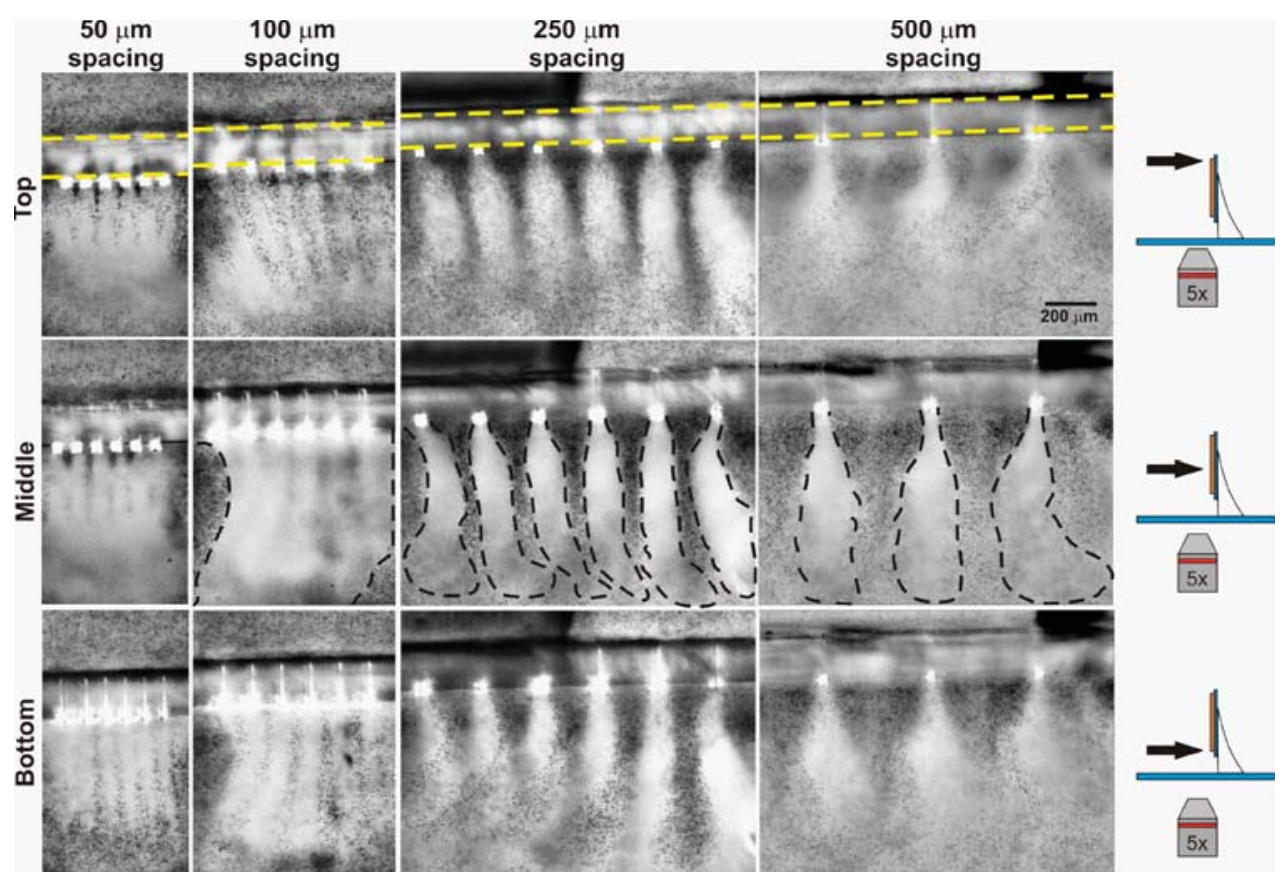

Figure 9: Nafion strips spaced at $50 \mu \mathrm{m}, 100 \mu \mathrm{m}, 250 \mu \mathrm{m}, \& 500 \mu \mathrm{m}$ edge-to-edge. Exclusion zones form from the Nafion surfaces and merge into an unbroken EZ in 50- and 100 micronspaced lines. Nafion strips are seen as the bright white squares on the images. At 250 and 500 microns, the EZ forms in discrete zones that do not combine. The yellow dashed lines demarcate edges of glass support. Dashed black lines indicate extent of clear EZ. Diagram on the far right indicates the viewing height (arrow) on which the objective was focused. 
The application of soft lithography techniques allowed for the deposition of rectangular strips of EZ-nucleating surfaces at discrete separations to assess whether EZs could combine to generate a continuous exclusion zone. We found that EZs generated from different nucleating surfaces can bond or anneal with one and other. A critical parameter that determines this interaction is the distance separating the nucleating strips.

Separations of $100 \mu \mathrm{m}$ or less between Nafion strips produced continuous EZs. Those EZs could extend, perpendicular to the surface of the glass coverslip, up to $1 \mathrm{~mm}$ away from the surface. Separations more than $100 \mu \mathrm{m}$ resulted in exclusion zones that did not combine into a continuous EZ, but produced several independent EZs (Fig. 9). The independent EZs were stable, long lasting (several hours) and extended 400-600 $\mu \mathrm{m}$ away from the glass surface. In the $50 \mu \mathrm{m}$ and $100 \mu \mathrm{m}$ lines that EZs did combine, the extent of the EZ (perpendicular to the glass surface) was larger than those of the non-combined EZs.

An obvious question to ask is the role of separation between EZ-generating surfaces. Why is the separation critical? The rectangular Nafion strips are exposed to water; but they are also surrounded by the glass surface. The faces abutting the glass surface are not seen to generate visible EZs in the $250 \mu \mathrm{m}$ and $500 \mu \mathrm{m}$ strips (Fig. 9, middle row, left columns), but the $50 \mu \mathrm{m}$ and $100 \mu \mathrm{m}$ strips show a stable EZ (middle row, right columns). If EZ formation occurs by epitaxial growth [16], then that growth could be disrupted by the nearby glass surface. EZs require water to build their structure and the glass surface may act as a barrier, preventing the EZ from structuring water molecules.

On the other hand, the situation differs at positions far from the glass surface. If an EZ can grow and interact with another EZ, then a stable structure is achieved and no more water need be recruited to help build the EZ at that location. An analogy to this observation is the construction of a brick wall. Once a brick is laid down, it remains there; only new bricks need be added on top to build the wall. Growth occurs only at the top, leading edge of the wall. In the case of the lateral growth, this is akin to building an arched brick wall. If the distances are short enough, the arches can meet and form a stable structure; if they are too long, the arch collapses.

A final observation made during the experiments was the phenomenon of EZ-induced microsphere flow. Microspheres that had settled onto the floor of the chamber were seen to migrate along the floor, at a constant velocity, in the direction of the glass surface that contained the Nafion. They flowed until approaching the edge of the Nafion-generated EZ, where they finally accumulated (Fig. 10). This accumulation was continuous and could be seen to attract all microspheres in the chamber even at 72 hours after the start of the experiment.

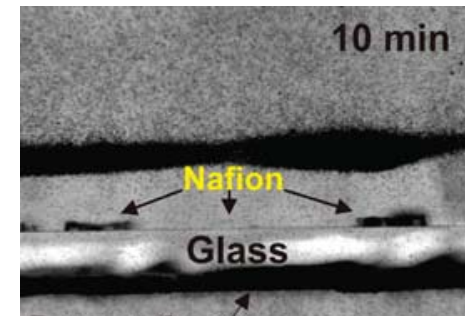

Bare surface

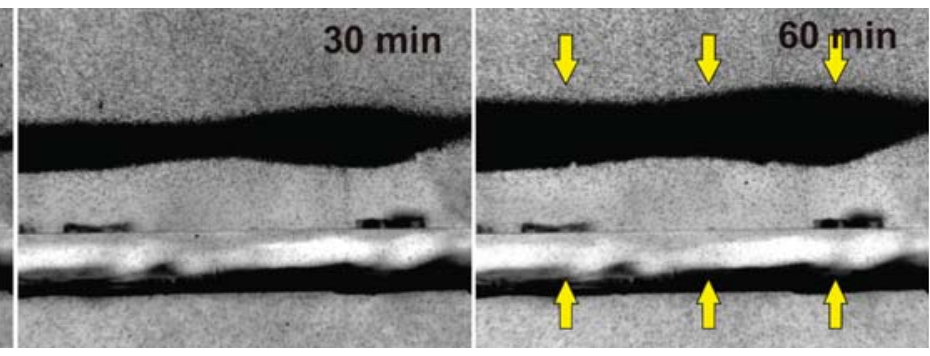

Figure 10: A vertically positioned Nafion coated glass coverslip section induces microsphere accumulation. Time lapse imaging reveals the steady migration and accumulation of microspheres at the bottom of the observation chamber. Yellow arrows indicate the direction of microsphere movement. Microspheres are $2 \mathrm{~mm}$ diameter carboxylate. 

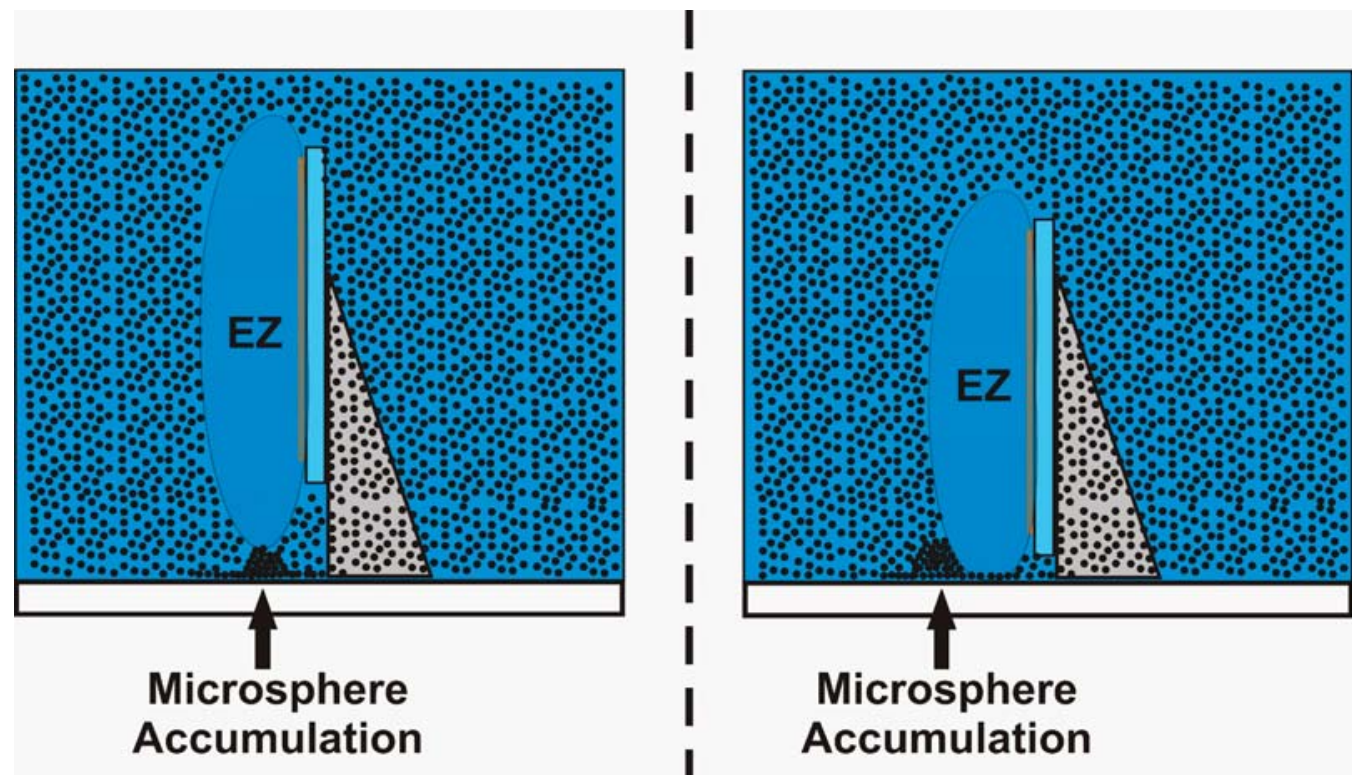

Figure 11: Diagram of EZ mediated microsphere exclusion and accumulation. Left, Nafion coated glass is held upright. Bottom is at a distance more than 200 microns from the bottom. Microspheres accumulate at the very bottom edge of the EZ. Right, Nafion coated glass is held upright 20 to 50 microns from the bottom. Microspheres accumulate at the distal end of the EZ.

The deposition site depended on the height of the bottom of the Nafion-containing glass (Fig. 11). If raised past a critical height $(\sim 200 \mu \mathrm{m})$, the accumulation occurred closer to Nafion surface (Fig. 11, left). Conversely, if the bottom was lower $(\sim 20-50 \mu \mathrm{m}$ from chamber floor), then the microspheres would accumulate at the far edge of the exclusion zone (Fig. 11, right).

This is not the first time that this accumulation phenomenon has been observed. Zhao et al. (in press) have noted that microspheres beyond the exclusion zones adjacent to resin beads produce accumulations similar to those reported here. It appears that fluid flow and material accumulation at the edge of the EZ are general phenomena associated with EZs. The mechanism for this phenomenon remains unclear, but might be related to attractions generated by separated charges (Zhao et al. in press).

In conclusion, Nafion strips on a glass substrate generate EZs that can combine with other EZs as long as the distances separating the strips are less than approximately $100 \mu \mathrm{m}$. The demonstrated ability of EZs to combine implies that continuous EZs can be created by using minimal amounts of starter material spaced judiciously. This feature has direct implications for water purification technology [7], as well as a potentially new understanding for the role of proteins at the lipid bi-layers of cells. The unstirred water layer [12], a water region $10-50 \mu \mathrm{m}$ next to the exterior of the cell surface that acts as a diffusion barrier for certain solutes, could also be explained by the EZ generating properties of certain proteins at the cell surface. If proteins (or groups of proteins) can generate individual EZs that can combine, then the unstirred layer could prove to be the biological application of the general EZ phenomenon. 


\section{REFERENCES}

[1] Zheng, J.M. \& Pollack, G.H., Long-range forces extending from polymer-gel surfaces. Phys Rev E Stat Nonlin Soft Matter Phys, 68(3 Pt 1), 031408, 2003. doi:http://dx.doi.org/10.1103/ PhysRevE.68.031408

[2] Zheng, J.M., Chin, W.C., Khijniak, E., Khijniak, E., Jr. \& Pollack, G.H., Surfaces and interfacial water: evidence that hydrophilic surfaces have long-range impact. Adv Colloid Interface Sci, 127(1), pp. 19-27, 2006. doi:http://dx.doi.org/10.1016/j.cis.2006.07.002

[3] Klimov, A. \& Pollack, G.H., Visualization of charge-carrier propagation in water. Langmuir, 23(23), pp. 11890-11895, 2007. doi:http://dx.doi.org/10.1021/la701742v

[4] Ovchinnikova, K. \& Pollack, G.H., Can water store charge? Langmuir, 25(1), pp. 542-547, 2009. doi:http://dx.doi.org/10.1021/la802430k

[5] Zhao, Q., Ovchinnikova, K., Chai, B., Yoo, H., Magula, J. \& Pollack, G.H., Role of proton gradients in the mechanism of osmosis. J Phys Chem B, 113(31), pp. 10708-10714, 2009. doi:http://dx.doi.org/10.1021/jp9021568

[6] Zheng, J.M., Wexler, A. \& Pollack, G.H., Effect of buffers on aqueous solute-exclusion zones around ion-exchange resins. J Colloid Interface Sci, 332(2), pp. 511-514, 2009. doi:http:// dx.doi.org/10.1016/j.jcis.2009.01.010

[7] Klyuzhin, I., Symonds, A., Magula, J. \& Pollack, G.H., New method of water purification based on the particle-exclusion phenomenon. Environ Sci Technol, 42(16), pp. 6160-6166, 2008. doi:http://dx.doi.org/10.1021/es703159q

[8] Zhao, Q., Zheng, J., Chai, B. \& Pollack, G.H., Unexpected effect of light on colloidal crystal spacing. Langmuir, 24(5), pp. 1750-1755, 2008. doi:http://dx.doi.org/10.1021/la703387m

[9] Chai, B., Yoo, H. \& Pollack, G.H., Effect of radiant energy on near-surface water. J Phys Chem $B$, 113(42), pp. 13953-13958, 2009. doi:http://dx.doi.org/10.1021/jp908163w

[10] Ovchinnikova, K. \& Pollack, G.H., Cylindrical phase separation in colloidal suspensions. Phys Rev E Stat Nonlin Soft Matter Phys, 79(3 Pt 2), 036117, 2009. doi:http://dx.doi.org/10.1103/ PhysRevE.79.036117

[11] Choi, D., Lee, W., Park, J. \&Koh, W., Preparation of poly(ethylene glycol) hydrogels with different network structures for the application of enzyme immobilization. Biomed Mater Eng, 18(6), pp. 345-356, 2008.

[12] Loftsson, T. \& Brewster, M.E., Physicochemical properties of water and its effect on drug delivery. A commentary. Int J Pharm, 354(1-2), pp. 248-254, 2008. doi:http://dx.doi.org/10.1016/j. ijpharm.2007.08.049

[13] Unger, E., Littlefield, J. \& Gado, M., Water content and water structure in CT and MR signal changes: possible influence in detection of early stroke. AJNR Am J Neuroradiol, 9(4), pp. 687-691, 1988.

[14] Rettig, J. \& Folch, A., Large-Scale single-cell trapping and imaging using microwell arrays. Anal. Chem., 77(17), pp. 5628-5634, 2005. doi:http://dx.doi.org/10.1021/ac0505977

[15] Chai, B.H., Zheng, J.M., Zhao, Q. \& Pollack, G.H., Spectroscopic studies of solutes in aqueous solution. J Phys Chem A, 112(11), pp. 2242-2247, 2008. doi:http://dx.doi.org/10.1021/ jp710105n

[16] Roy, R., Tiller, W.A., Bell, I. \& Hoover, M.R., The structure of liquid water; novel insights from materials research; potential relevance to homeopathy. Materials Research Innovations Online, 9(4), 32, 2005. 